Thorax (1974), 29, 271.

\title{
Diffuse pulmonary alveolar fibrosis ${ }^{1}$
}

\author{
JOHN GUYETT SCA D I NG ${ }^{2}$
}

Brompton Hospital and Hammersmith Hospital, London

\begin{abstract}
Scadding, J. G. (1974). Thorax, 29, 271-281. Diffuse pulmonary alveolar fibrosis. The problems of diagnostic categorization of diffuse pulmonary alveolar fibrosis are outlined, and attention is drawn to the different implications of categories defined aetiologically and histopathologically. The pattern of pulmonary insufficiency associated with changes of these sorts is described, and the importance of using a terminology of pulmonary insufficiency which refers unequivocally to disorders of function is emphasized. The usage of the word 'interstitial' in the context of inflammations of the lung is critically examined. It seems to be used to imply predominant involvement of alveolar walls, which in other contexts would be regarded as 'parenchyma'. This unresolved conflict with accepted anatomical usage can be avoided by general acceptance of complementary conventions that 'pneumonia' refers to inflammations of the lung characterized by exudation into alveolar spaces leading to consolidation, and 'alveolitis' to those affecting predominantly alveolar walls. Some cases of alveolitis, so defined, can be shown to be due to reactions with inhaled organic dusts in the gas-exchanging part of the lungs in specifically sensitized individuals, constituting a group of aetiologically defined categories 'extrinsic allergic alveolitis'. A few are due to ingested toxic substances. Many remain of unknown cause and show a strong and early tendency to alveolar wall fibrosis: they constitute a histopathologically defined category 'cryptogenic fibrosing alveolitis'.
\end{abstract}

By 'diffuse pulmonary alveolar fibrosis' I mean widespread fibrosis in that part of the lungs, beyond the terminal bronchioles, which is concerned with gas exchange. This may result from a wide range of morbid processes, some of known and some of as yet unknown cause. I considered the problems of diagnosis presented by patients with such changes, with special reference to lung biopsy, in the Tudor Edwards Memorial Lecture three years ago (Scadding, 1970) and, earlier, with special reference to radiology (Scadding, 1952, 1956). In this lecture I propose to concentrate upon three respects in which knowledge relevant to these problems has advanced in the past 25 years. These are the delineation of the characteristic pattern of respiratory dysfunction; the elucidation of the importance of inhaled organic dusts in causing changes that may lead to fibrosis through antigen-antibody reactions in the gasexchanging part of the lungs; and the description and definition on histopathological grounds of a

${ }^{1}$ The Lumleian Lecture delivered at the Royal College of Physicians of London on 7 November 1973

${ }^{2}$ Emeritus Professor of Medicine, University of London group of cases of alveolar fibrosis developing from inflammatory processes of undetermined cause affecting predominantly alveolar walls. But before proceeding to these topics, I will attempt to place them in context by presenting a brief list of diagnostic categories of diffuse pulmonary alveolar fibrosis (Table). This is not intended to be exhaustive but to outline a logical approach to the problem of diagnosis.

\section{DIAGNOSTIC CATEGORIZATION}

The desirable end-point of the diagnostic process is knowledge of causation. If this is attained, the process is regarded as satisfactorily completed, and a diagnosis of a disease defined aetiologically will be made. But if, as often happens, this cannot be attained and the process stops at some stage short of causation, its end-point will consist in the diagnosis of a disease defined in terms of what is thought to be the most useful kind of knowledge that has been obtained (Scadding, 1959, 1967a, 1972). In diffuse alveolar fibrosis, this is generally thought to be that derived from histopathology. 
T A B L E

DIAGNOSTIC CATEGORIES OF PULMONARY ALVEOLAR FIBROSIS

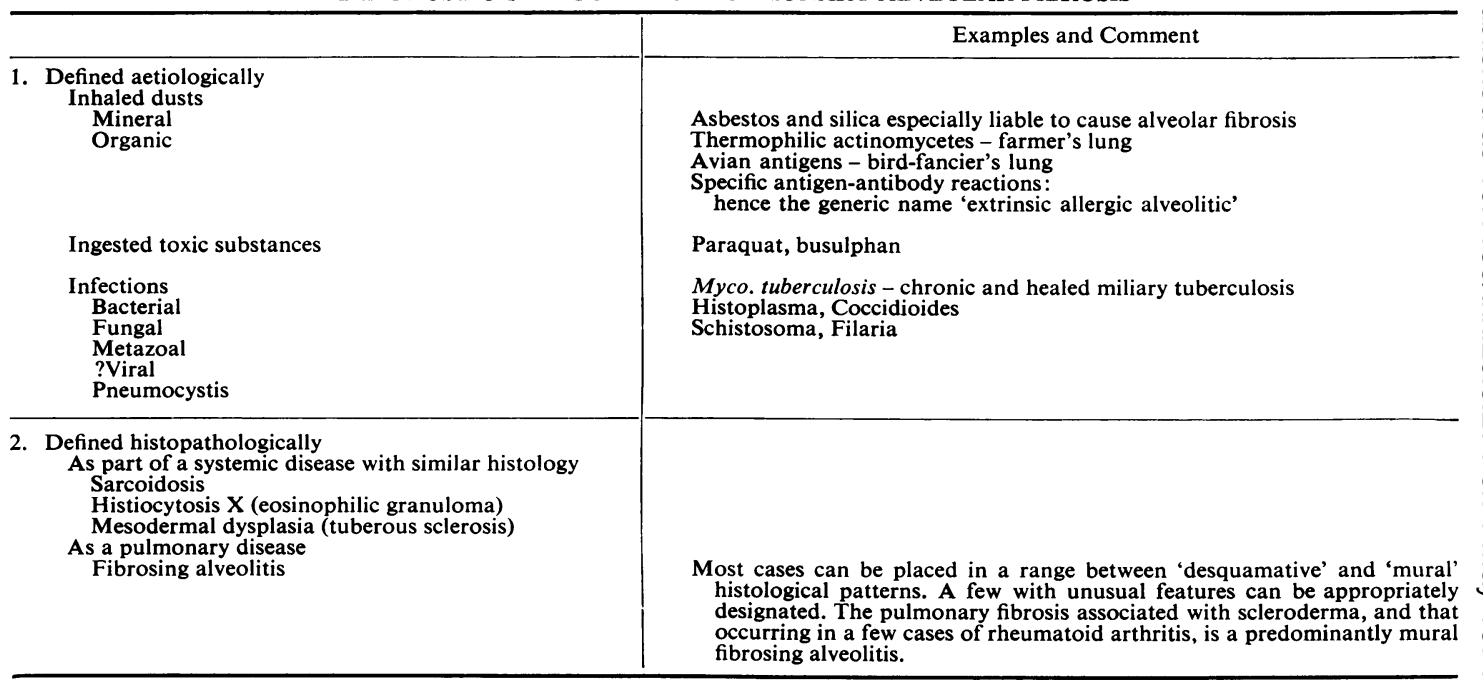

The diagnostic categories into which cases of widespread pulmonary alveolar fibrosis may be placed therefore fall into two groups-those defined in terms of aetiology, and those defined in terms of histopathology. Among those defined aetiologically, histopathology may, of course, provide a strong clue to causation but cannot usually establish it beyond doubt and may be equivocal. Those categories defined histopathologically and of undetermined cause can be subdivided into two subgroups-those in which the histopathological changes in the lungs are part of a systemic disease in which similar changes involve other organs, and those in which the defining histopathological changes are found only in the lungs, though there may be changes in other organs having a different pattern.

A list of this sort does not constitute a logically rigorous classification since the categories in its two parts are defined by criteria derived from different fields of study; there is thus no a priori reason why in all instances a category from the first part and a category from the second should be mutually exclusive. At first sight it might seem possible to convert the list into a classification by labelling its second part 'of unknown aetiology', thus making it the residual category of an aetiological classification. But this would not obviate all difficulties. No difficulty arises if we can be certain that no agent or factor which has been used as the defining characteristic of an aetiologically defined category can possibly be concerned in any case relegated to the residual category. We should, of course, be entitled to assume that a person making a diagnosis from this residual category has sought and failed to find evidence of any known cause; and if in future a group of cases is found to be associated with a hitherto unidentified agent, a new aetiologically defined category will be created for it. But there are two situations in which cases that are in fact associated with known agents may incorrectly be classified as 'of unknown aetiology'. A known agent may have been concerned in the past but is no longer present; or it may in fact be present but has not been detected by the methods used. I have discussed in connection with sarcoidosis the difficulties that arise from including 'unknown aetiology' in the definition of a disease; if knowledge is lacking or incomplete, a statement to this effect is more appropriately included in the description of the disease than in its definition (Scadding, 1967b). These considerations may be relevant to fibrosing alveolitis as well as to sarcoidosis. If a case is assigned to a histopathologically defined category, it can be assumed that no causal agent has been identified if none is mentioned. If, for any reason, it is thought advisable to emphasize this lack of knowledge, the word 'cryptogenic' or the phrase 'of unknown cause' can be added. But if in any case there is evidence suggesting that a known agent or factor may be concerned in causation, it should be possible to discuss its implications without the need to doubt the correctness of histopathological categorization. We must thus accept the need to forego a logically rigorous 
classification and resign ourselves to a list composed of two partial classifications, one aetiological and the other histopathological, which are not in principle mutually exclusive.

\section{PATTERN OF FUNCTIONAL IMPAIRMENT}

The pattern of pulmonary insufficiency caused by widespread changes in the peripheral gasexchanging part of the lungs was outlined by Baldwin, Cournand, and Richards (1948) as a combination of restrictive ventilatory insufficiency with insufficiency of alveolo-respiratory function characterized clinically by hypoxia and hyperventilation. Although both these aspects of function have been analysed in greater detail since then, this brief statement needs no important modification. The more complete description by Austrian et al. (1951) introduced the term 'alveolar capillary block'. This almost irresistibly suggests some sort of impediment to diffusion as the leading component of this sort of dysfunction. In fact, in most situations impairment of diffusion through alveolar membranes is only a minor factor, the major part of the impairment of 'alveolo-respiratory function' being contributed by abnormal variations in ventilation-perfusion ratios in different groups of alveoli, and some by diminution of alveolar capillary blood volume (West, 1965). The term 'gas transfer' was first suggested in relation to tests such as those based upon the uptake of carbon monoxide, which are affected by all these factors (Cotes, 1963). It is now widely used for those aspects of pulmonary function which Baldwin et al. (1948) called 'alveolorespiratory', and has the advantage of avoiding the use of such a general term as 'respiratory' in a specialized sense. It may be regarded as referring to the resultant of all the factors concerned in the transfer of gases between air-spaces and pulmonary capillary blood throughout the lungs.

It is unfortunate that several points emphasized by Baldwin et al. have been largely disregarded. They urged that 'every clinical case is an example of both ventilatory and alveolo-respiratory forms of insufficiency'; and they used 'restrictive' to refer specifically to a form of ventilatory insufficiency. In spite of this, the term 'restrictive lung disease' has crept into rather wide use to refer to a combination of impaired gas transfer and restrictive ventilatory insufficiency. This is regrettable. It takes no cognizance of the importance of referring separately to these two aspects of functional impairment which occur in varying proportions from case to case. It frustrates the attempt to introduce into clinical practice the use of terms referring unequivocally to defined patterns of respiratory dysfunction without commitment to implications about structural changes or causation, thus permitting unhampered discussion of correlations (Scadding, 1966). The awkwardness of such terms as 'restrictive lung disease' and 'obstructive airways disease' is made apparent if they are compared with oliguria, raised intracranial pressure, congestive heart failure, biliary obstruction, and many others which refer, and are shown by their verbal form to refer, to a disorder of function which can be correlated with a variety of structural and causal aspects of a case. If anyone spoke of 'congestive failure heart disease' or 'raised intracranial pressure brain disease', he would certainly be asked what contribution the word 'disease' was making, and its needless addition would justly be regarded as obscurantist verbiage. No one thinks it necessary to add 'disease' to pulmonary hypertension: why do some people feel unhappy without this word when they refer to the ventilatory rather than the circulatory function of the lungs?

As in other contexts where analysis and quantification in the laboratory have introduced precision into clinical observation, the pattern of functional disturbance produced by widespread changes in the gas-exchanging part of the lungs can be at least suspected and, when fully developed, confidently recognized in the course of a careful clinical examination. It can be confirmed and quantified by relatively simple methods. The cardinal symptom is dyspnoea on exertion, without wheeze. This is associated with greater pulmonary ventilation than would be expected from the level of physical activity, so that the patient may appear to be overbreathing at rest, and even more on exertion. In the more severe cases there is obvious tachypnoea. Typically, there is little or no evidence of airways obstruction, though in some instances the cause of the alveolar changes may also affect the airways; and in a cigarettesmoking population the concurrence of an unrelated chronic obstructive bronchitis must be expected in an appreciable minority of cases.

If auscultatory signs are present in the chest, they consist in discontinuous sounds (crepitations) rather than wheezes. In severe cases, cyanosis may be evident, in some only after exertion. The ventilatory defect can be quantified by spirometry: the vital capacity is reduced, but in a forced expiration a normal or higher than normal proportion of it is expelled in the first second, and expiratory 
flow-rate may be normal. The high minute ventilation can be confirmed by spirometer or gasmeter. Measurement of arterial blood gases serves to confirm, and to provide some quantitative information about, the impairment of gas transfer. Arterial oxygen pressure $\left(\mathrm{PaO}_{2}\right)$ at rest may in a mild case be normal, but in others will be below normal, and in all cases will fall on exercise; and arterial carbon dioxide pressure $\left(\mathrm{PaCO}_{2}\right)$ is on the low side of, or below, the normal range, rising only in the terminal stages of the disease. Thus, clinical findings and the results of spirometry and blood gas estimations provide the basis for a confident opinion that the peripheral gas-exchanging part of the lung is widely involved in some disease process.

Further laboratory studies can add more detailed information but are not essential for diagnosis. Estimation of the transfer factor for carbon monoxide is a convenient way of quantifying in a single variable the complex set of factors concerned with gas transfer, and in practice its principal use is in following the progress of a patient. Total lung capacity is likely to be below the predicted level; in many cases, the small area of the lung fields in a chest radiograph known to have been taken in full inspiration permits a reasonable deduction that the lungs are small. The reduced total lung capacity tends to be normally subdivided, with proportionate reductions in residual volume and vital capacity, in the absence of complicating factors. Measurement of intrathoracic pressure with an oesophageal balloon provides additional information about lung compliance, which is likely to be diminished, especially if the alveoli are extensively involved in a densely fibrotic process. Thus the complete pattern can be elucidated. It can be summarized as small stiff lungs with normal airways, leading to a restrictive ventilatory defect, and disorganization of that part of the lung where normally there is an orderly and well balanced encounter between gas in alveoli and blood in alveolar capillaries, leading to impaired gas transfer.

\section{ALVEOLITIS}

Just over nine years ago, I suggested (Scadding, 1964) that the term 'fibrosing alveolitis', which I had been using for some time in verbal discussions and had found convenient, might be appropriate as a generic name for the disease that had been described under such names as interstitial pneumonia or pneumonitis and chronic Hamman-Rich disease or syndrome (Grant, Hillis, and Davidson,
1956; Rubin and Lubliner, 1957; Scadding, 1960; Livingstone, Lewis, Reid, and Jefferson, 1964). This term has been adopted fairly widely in Great Britain (e.g., Crofton and Douglas, 1969) but not on the other side of the Atlantic, and the resulting duality of terminology certainly gives rise to occasions of confusion. Perhaps it will be helpful to reiterate my reasons for the avoidance of the word 'interstitial' in this context, and for suggesting that the adoption of a suitable convention about the usage of the word 'alveolitis' makes this possible.

The difficulty about 'interstitial' in relation to the lungs is that it has been used in different and $\dot{\omega}$ even contradictory senses, so that it is now impossible to define it in any way that is not either incompatible with one or more of its current usages, or so general as to be virtually meaningless. Anatomically, the interstitial tissue of the lung has been described as constituted by the peribronchial, perivascular, interlobular, and subpleural connective tissue (von Hayek, 1953, 1960). This accords with usage in relation to other organs, such as the liver, the kidneys and glands, where the supporting connective tissue is referred to as interstitial, as opposed to the functionally specific components which are said to constitute the parenchyma. In the liver and in secreting glands this distinction is fairly easily drawn. But in the lungs it is difficult to define a functionally specific part: airways, blood vessels, and alveoli all play essential roles. Thus there is no precise definition of pulmonary parenchyma, and it is not surprising that hopeless confusion surrounds the use of this term. The most frequent use of it, certainly by physicians and radiologists, appears to be to refer to the alveoli as opposed to major airways and blood vessels. But this is the very part of the lung involved in so-called 'interstitial' fibrosis, which 을 notably does not involve the major airways or the large blood vessels, or the connective tissue sheaths around them that constitute a large part $N$ of the anatomical interstitial tissue.

Perhaps we should give up the attempt to maintain complementary meanings of 'parenchyma' $\mathrm{W}$ and 'interstitium' in relation to the lungs and attempt to define these terms independently of $\stackrel{C}{C}$ each other. 'Parenchyma' seems to be beyond $\Phi$ redemption. Can anything be done for 'inter-? stitial'? Technical usages should be at least con- $\frac{T}{3}$ cordant with dictionary definitions, though they $\stackrel{\circ}{\mathbb{D}}$ generally need redefinition in more precise and $\stackrel{\odot}{\odot}$ often conventionally restricted terms. The Shorter $\stackrel{\mathbb{Q}}{\square}$ Oxford English Dictionary defines interstitial, 우 when used of a morbid process, as 'taking place in 
the interstices of a body' and interstices as 'relatively small or narrow spaces between parts of a body'. Evidently, the user of the word 'interstitial' must be prepared to say between what structures are situated the small spaces to which he is referring. In some conţexts, e.g., in speaking of interstitial fluid, I suppose the reference is to spaces between cells: and here it would be clearer to use the word 'intercellular'. But if 'interstitial' refers generally to spaces between cells, it can be applied to any sort of fibrosis, since presumably it must be in the spaces between cells that fibroblasts lay down reticulin and collagen. Perhaps, in relation to fibrosis in the lungs, 'interstitial' is intended to specify a process within alveolar walls rather than organization of exudate in alveolar spaces. But here is confusion worse confounded. If the alveolar walls are interstitial, between what are they? They are bounded on both sides by alveolar spaces: and so we are in the absurd position of referring to spaces between spaces! It is interesting that Liebow (1968), who has made valuable contributions to the histopathology of what he calls 'interstitial pneumonias', admits that 'this is a blanket term for any tissue response in the lung which takes place predominantly in the supporting structures rather than within alveoli'. But alveolar walls, or even the spaces between their cells, can hardly be called supporting structures. I conclude that in relation to the lung 'interstitial' is also past redemption, and consequently I have abandoned the use of it.

What is required is evidently a term that can be used to refer to inflammatory changes predominantly affecting alveolar walls, as opposed to 'pneumonia', which is generally used to refer to inflammations of the lung characterized by consolidation by inflammatory exudation filling alveolar spaces. If this restriction of meaning of 'pneumonia', which is often implied, were explicity stated and generally agreed, the word 'alveolitis' could be adopted, with a complementary restriction, to refer to inflammations of the lungs affecting predominantly alveolar walls. A pneumonia usually resolves, but if it does not it may lead to fibrosis by organization of intraalveolar exudate, presenting an easily recognizable histopathological pattern. If fibrosis develops in the course of an alveolitis, it appears within alveolar walls. The general acceptance of these easily explained verbal conventions would remove the need for the use of the word interstitial, which we have seen it is impossible to define clearly, in relation to pulmonary alveolar fibrosis.

\section{EXTRINSIC ALLERGIC ALVEOLITIS}

The role of reactions to inhaled organic dusts in the peripheral gas-exchanging part of the lungs as a cause of alveolar fibrosis was first elucidated as a result of studies of 'farmer's lung'. This was first described as an occupational disease of farmers exposed to dust from mouldy hay in 1932 (Campbell, 1932) and has now been found in many areas in which farming practices and climatic conditions are such that exposure to this dust occurs (Fuller, 1953; Dickie and Rankin, 1958; Staines and Forman, 1961). At first it was tentatively regarded as a pulmonary mycosis (Fawcitt, 1936; Törnell, 1946). But it was not until the immunology of reactions to inhaled organic dusts was elucidated (Pepys, Riddell, Citron, and Clayton, 1962; Pepys, Longbottom, and Jenkins, 1964), and it was shown that inhalation of mouldy hay extracts reproduced the features of the acute episodes of the disease in affected farmers (Williams, 1961, 1963 a and b) that its pathogenesis was understood. It is now evident that 'farmer's lung' is caused by specific antigen-antibody reactions in the peripheral gasexchanging parts of the lungs, precipitating antibody being an important part of the host reaction, and the thermophilic actinomycetes, which are the predominant moulds in hay that has become over-heated in process of going mouldy (Corbaz, Gregory, and Lacey, 1963; Gregory and Lacey, 1963), being the source of the antigen (Pepys et al., 1963; Gregory et al., 1964). Diseases due to similar reactions to other inhaled organic dusts have been described, and usually named after the occupation in which the patient has been exposed to the causative dust, e.g., bird-fancier's lung (Barboriak, Sosman, and Reed, 1965; Hargreave, Pepys, Longbottom, and Wraith, 1966), mushroom grower's lung (Sakula, 1967), and malt-worker's lung (Riddle et al., 1968). A term by which reference can be made to the whole group of diseases caused by antigen-antibody reactions to inhaled dusts in the gas-exchanging part of the lungs is evidently required. 'Extrinsic allergic alveolitis' (Pepys, 1967) meets this need. It has been criticized on the grounds that in some cases the functional defect includes an element of airways obstruction, and bronchioles as well as alveoli are involved in the histopathological changes (Hapke et al., 1968). But the alveoli are at least an important site of the reaction, which in them is an alveolitis, as defined above, rather than a pneumonia; so that although I should regard 'extrinsic allergic bronchiolo-alveolitis' as more comprehensively indicative of the extent of the changes 
(Scadding, 1970), the shorter name seems sufficient, provided that it is not taken to imply that the changes are confined to alveoli.

As would be expected, the clinical picture depends upon the severity, duration, and periodicity of exposure, and to some extent upon the nature of the dust. Intermittent exposure to heavy concentrations causes, after an interval of several hours, headache, fever, and tightness in the chest: this tends to occur in farmers after such activities as breaking up mouldy bales of hay, or in pigeonfanciers after cleaning out the loft. Severe exposure may result in several days of illness and transient widespread mottling in the chest radiograph. Although complete recovery is the rule after a limited number of mild exposures, fibrosis may develop after severe exposure or after repeated exposures. Persistent exposure to small amounts of dust, as in people who keep budgerigars inside their homes, leads to the insidious development of fibrosis; affected individuals seek advice only after they have become breathless with established fibrosis, which tends to affect the upper parts of the lungs and may cause radiographic changes resembling those of some very indolent forms of tuberculosis. In severely affected patients, and especially in those exposed to avian antigens, there may be evidence of airways obstruction as well as restrictive ventilatory defect and impairment of gas transfer. We have here an excellent illustration of the importance of using a terminology of pulmonary insufficiency which, in the words of Baldwin et al. (1948), 'permits quantitative analysis of any given case'.

Histopathology in an active stage shows distinctive changes (Totten, Reid, Davis, and Moran, 1958; Seal et al., 1968). There is patchy cellular thickening of alveolar walls, including some histiocytes; some involvement of bronchioles; more or less well-formed epithelioid cell granulomas in both these sites; giant cells in variable number, some containing lanceolate vacuoles; lymph follicle hyperplasia; and variable numbers of plasma cells. But as the condition becomes more chronic, and after exposure ceases, the active inflammatory and granulomatous elements diminish, and the end-stage of fibrotic honeycombing may be indistinguishable from that of a cryptogenic fibrosing alveolitis. Thus the contribution of histopathology to diagnosis varies with the stage of the disease. Even in the active stage, when the appearances are almost diagnostic, the diagnosis depends in the final analysis upon clinical and immunological findings.
Immunologically, precipitating antibody to $\stackrel{\overrightarrow{\mathrm{F}}}{\rightarrow}$ antigens contained in the relevant dust can be $\overrightarrow{0}$ demonstrated in the blood serum. This finding confirms that exposure sufficient to stimulate the $\frac{\bar{D}}{\frac{D}{}}$ formation of antibodies has occurred: this does $\stackrel{\mathbb{Q}}{\Omega}$ not, of course, prove that lung disease is caused by this exposure, though it gives support to this hypothesis. In some sorts of extrinsic allergic alveolitis, e.g., those associated with exposure to $\vec{\omega}$ avian antigens, but not in farmers, skin tests give $\stackrel{\sigma}{\circ}$ dual reactions-an immediate weal-and-flare, $\overrightarrow{\vec{x}}$ which subsides and is followed in about six hours iु by a diffuse erythematous swelling. Challenge by $\dot{\omega}$ inhalation of an aerosol containing the offending is antigen causes both systemic and pulmonary re- actions, starting after about six hours. The sys- 은 temic reaction consists in headache, slight fever, $\overrightarrow{-}$ tachycardia, and neutrophil leucocytosis; the local reaction in a sensation of tightness in the chest, crepitations at the lung bases, and diminution in lung compliance and in gas transfer factor. Such tests should be performed only if essential for diagnosis. They should be initiated with caution, using a small challenge and repeating with a larger one if necessary after two or three days.

In all cases of alveolar fibrosis the possibility of extrinsic allergic alveolitis should be considered. The diagnosis depends upon a history of exposure to an organic dust and demonstration of specific immunological reactivity to antigens in the suspected dust. Recognition of the clinical syndrome may lead to the discovery of an unsuspected dust hazard. This may be unsuspected exposure to a dust known to be associated with extrinsic allergic alveolitis as, for example, when the growth of thermophilic actinomycetes in the humidifying element of an air-conditioning system was found to be causing office workers to suffer from extrinsic allergic alveolitis, identical with farmer's lung (Banaszak, Thiede, and Fink, 1970). Or, of course, it may be exposure to a dust not previously identified as a cause of extrinsic allergic alveolitis.

\section{DIFFUSE FIBROSING ALVEOLITIS}

The diagnosis of diffuse fibrosing alveolitis implies that the lungs are known or believed to be widely affected by an alveolitis, with developing or established fibrosis within alveolar walls. In many cases conforming to this description, the cause of this change remains unknown, and these can be specified as 'cryptogenic' or 'of unknown cause'. Such cases vary both in details of histopathology and in the clinical picture. The histopathology can best 
be described in a range between two polar patterns, which may be called 'desquamative' and 'mural' (Scadding and Hinson, 1967). The desquamative pattern is characterized by the presence of large numbers of mononuclear cells, many resembling granular or type II pneumocytes, within alveolar spaces; relatively little inflammatory thickening or fibrosis in alveolar walls, though proliferation of reticulin is conspicuous in appropriately stained sections; and maintenance of the alveolar pattern of the lung. At the other end of the range, the mural pattern is characterized by the prominence of alveolar wall thickening and fibrosis, often patchy in distribution and distorting alveolar structure; and within the alveoli, fewer cells of more varied types, including phagocytic rather than granular pneumocytes, lymphocytes, plasma cells, and granulocytes.

Most cases of cryptogenic fibrosing alveolitis show features intermediate between these two patterns; and in a few, characteristic examples of the desquamative and the mural patterns have been found in the same lung. Lymphoid follicle hyperplasia may be observed in association with either of these patterns but tends to be more prominent at the desquamative end of the range. Where there is much fibrosis, and hence the pattern is by definition mural, obliteration of groups of alveoli in confluent fibrosis and conversion of others into small cyst-like spaces lined by cuboidal epithelium may produce a honeycomb-like appearance, both radiologically and on the cut surface of the lung at necropsy. This is usually most conspicuous in the costophrenic fringe of the lower lobes.

Clinically, dyspnoea on exertion is the constant symptom. Most patients, and especially the older ones, develop symptoms insidiously, without much constitutional disturbance; in a few, mostly among the younger, the illness starts more rapidly, with fever in some. There is usually no evidence of involvement of airways, but in the early active stage and later with severe and extensive fibrosis cough, unproductive or with scanty mucoid expectoration, may be troublesome. Clubbing of fingers and toes occurs in a high proportion of cases and may be a very early symptom. In my own series of 124 patients, $70 \%$ showed clubbing on first examination. Among those in whom the histological pattern was established at this time, the proportion with clubbing was similar at the desquamative and the mural ends of the range. In this same group, there appeared to be some correlation between the patterns of histopathology and of functional defect: a desquamative pattern tended to be associated with a more prominent ventilatory component, and a mural pattern with a more prominent gas transfer component of the functional defect (Scadding and Hinson, 1967).

Radiological appearances, though variable, show some correlation with histopathological pattern. The desquamative pattern presents either with ill-defined patchy opacities, usually affecting the lower parts of the lungs most but sometimes irregularly distributed; or with bilateral basal shadowing above an elevated diaphragm, indicating contraction of the lower lobes. This latter appearance occurs also in some patients who show an intermediate or even a predominantly mural pattern. Most patients with mural pattern, short of honeycombing, show irregular small discrete opacities, usually most profuse in the lower parts of the lungs, but sometimes generalized. The association of a normal chest radiograph with fibrosing alveolitis of both desquamative (Liebow, Steer, and Billingsley, 1965; Scadding and Hinson, 1967) and intermediate (Scadding, 1970) patterns is well documented. The late honeycombed pattern is, of course, easily recognizable radiologically.

In the late fibrotic stage, bronchography shows considerable narrowing both of the peripheral unfilled zone, which corresponds to a mainly alveolar part of the lung, and of the adjacent zone, in which normally there is a fine pattern of bronchioles branching at intervals of a few millimetres. This gives the larger bronchi outlined by the contrast medium the appearance of extending much further towards the periphery of the lung than usual, suggesting at first sight dilatation (Scadding, 1960) but evidently due to the narrowing by fibrosis of the peripheral respiratory zone of the lung.

Fibrosing alveolitis may be associated with several sorts of extrapulmonary disease. About one in eight cases of fibrosing alveolitis in unselected series also have rheumatoid arthritis. In these cases the histological pattern has generally been found to be mural; there may also be evidence in places of focal organizing pneumonia. The course tends to be indolent, with slow progression, eventually resulting in some 'honeycombing'. In some cases, other changes known to be associated with rheumatoid arthritis-necrobiotic nodules in the pleura or in the subpleural part of the lungs, and pleural effusion-are also found (Scadding, 1969). The lung changes that occur in a proportion of patients with scleroderma (systemic sclerosis) are a very indolent fibrosing alveolitis of mural 
pattern, and may be accompanied by all sorts of combinations of the other organ manifestations of systemic sclerosis. I have observed three patients with fibrosing alveolitis who had Raynaud's phenomenon of recent onset with equivocal changes in the skin, which proved non-progressive, a sort of 'forme fruste' of scleroderma.

Fibrosing alveolitis, of all patterns between the desquamative and the mural, is accompanied in a proportion of patients by certain other diseases affecting single organs or tissues, and associated with auto-immune factors, e.g., Sjögren's syndrome, Hashimoto's thyroiditis, polymyositis (Scadding and Hinson, 1967), chronic active hepatitis (Turner-Warwick, 1968), and hyperglobulinaemic renal tubular acidosis (Mason, McIllmurray, Golding, and Hughes, 1970). Antinuclear factor (ANF) and rheumatoid factor (RF) are demonstrable in the blood serum in a higher proportion of patients with fibrosing alveolitis than of control groups; about one-third have ANF and one-third RF but only a few have both (Turner-Warwick and Doniach, 1965; TurnerWarwick and Haslam, 1971). The titres of both are usually low, except in those patients who also have rheumatoid arthritis and have the expected high titres of RF. The incidence of RF and ANF in patients with extrinsic allergic alveolitis is similar to that in controls. Among workers exposed to asbestos, those with asbestosis but not those with comparable exposure and no lung fibrosis have an incidence of RF and ANF comparable with that found in cryptogenic fibrosing alveolitis. This is of interest in view of the many similarities between asbestosis and cryptogenic fibrosing alveolitis, e.g., the frequency of finger clubbing, the progressive course after exposure has ceased, and the similar pattern of pulmonary insufficiency.

The group of cases categorized on histopathological grounds as diffuse fibrosing alveolitis is, of course, likely to be aetiologically heterogeneous. It seems probable that those that are associated with systemic diseases of unknown cause share some causal factor or factors with these, but the irregular occurrence of these associations suggests that this is unlikely to amount to aetiological identity. The cause of fibrosing alveolitis is just as unknown if it occurs in a patient with rheumatoid arthritis, or scleroderma, or Sjögren's syndrome, as if it occurs as a clinically lone phenomenon. Nevertheless, these associations suggest that the elucidation of the pathogenesis of these and related diseases might throw light also upon that of some cases of 'lone' fibrosing alveolitis.

The occurrence of familial cases of fibrosing alveolitis suggests that there is at least one group in which genetic factors are important (Donohue, Laski, Uchida, and Munn, 1959; Bonanni, Frymoyer, and Jacox, 1965; Koch, 1965; Adelman, Chertkow, and Hayton, 1966; Swaye, van Ordstrand, MacCormack, and Wolpaw, 1969; Wagley, 1972). These familial cases show some interesting features. Those in which information was available from biopsy or necropsy showed a histological pattern at the mural end of the range. In several of the familial aggregations, the disease appeared to be transmitted as an autosomal dominant trait (Bonanni et al., 1965; Swaye et al., 1969; Wagley, 1972). Although it might not become manifest until adult life, some members of affected families have developed it in childhood or even infancy; and the rate of progression has varied greatly from case to case. Pulmonary fibrosis does not seem to have been associated with systemic disease, such as rheumatoid arthritis, in affected members of these families. In one family, some unaffected members showed abnormalities of serum proteins (Bonanni et al., 1965). It seems possible that the familial cases constitute a pathogenetically distinct group.

The possibility that some cases at present cryptogenic may prove to be examples of hitherto unrecognized kinds of extrinsic allergic alveolitis has already been mentioned. Not only antigens as yet unidentified but also antigen-antibody reactions still to be elucidated may be concerned.

Prolonged administration of busulphan (Oliner, 3 Schwartz, Rubio, and Dameshek, 1961; Heard and Cooke, 1968; Littler, Kay, Hasleton, and Heath, ३ 1969); and of bleomycin (Fleischman et al., 1971; De Lena, Guzzon, Monfardini, and Bonadonna, 1972) may cause a pulmonary alveolar fibrosis, which includes some of the features seen in cryptogenic fibrosing alveolitis, particularly the of desquamation of type II pneumocytes into alveolar $N$ spaces. The lung changes in paraquat poisoning include fibrosing inflammatory thickening of alveolar walls (Bullivant, 1966; Matthew, Logan, o Woodruffe, and Heard, 1968). Thus the pos- $\frac{\Gamma}{\Phi}$ sibility that as yet unidentified ingested substances $\stackrel{\infty}{+}$ may be concerned in the causation of some cases 7 at present classified as cryptogenic fibrosing alveolitis must be borne in mind.

In the present state of knowledge, the question $\stackrel{\vec{\Phi}}{\varnothing}$ whether differences in histological pattern of cryptogenic fibrosing alveolitis can be correlated 
with differences in pathogenesis must be left open. The desquamative pattern corresponds with the 'desquamative interstitial pneumonia' of Liebow et al. (1965). They drew attention to the differences in clinical course and especially in response to corticosteroid treatment between this and other kinds of alveolar fibrosis, and claimed that it rarely progresses to a densely fibrotic pattern. But the clinical differences are those that would be expected from the criteria of histological selection: and a desquamative has been observed to progress to a mural pattern (Scadding and Hinson, 1967). In spite of this, there is no reason to suppose that a mural is necessarily preceded by a desquamative pattern, since a mural pattern may be found in what appears to be an early stage of a patient's illness. It is, of course, well recognized that there can be wide differences in histological reaction to the same causal agent, mycobacteria, especially in relation to the balance between cellular reaction and fibrosis. And in extrinsic allergic alveolitis, a similar pathogenesis underlies the slow development of fibrosis in budgerigar fanciers and the acute largely reversible reactions in farmers. For these reasons the histological patterns of cryptogenic fibrosing alveolitis should be named in terms that are evidently no more than descriptive; correlations with pathogenesis can be discussed profitably only when causal factors have been identified.

\section{CONCLUSION}

In this lecture I have discussed only some of the problems of diffuse pulmonary alveolar fibrosis. I have said little or nothing about some important groups of cases, e.g., those due to infections or to mineral dust, or those associated with granulomatous disease of unknown cause. Nor have I referred, save in passing, to such matters of concern to the clinician and his patient as prognosis and treatment. My concern has been not these obvious practical functions of the physician, but rather those activities which I believe to constitute his unique and essential scientific role. These are the integration of the information derived from all relevant disciplines with that derived from his own clinical skills to the understanding of the problems presented by individual patients; and clear definition of the diagnostic categories which are the basis of the disease terminology in which the results of this integration are expressed and discussed.

\section{REFERENCES}

Adelman, A. G., Chertkow, G., and Hayton, R. C (1966). Familial fibrocystic pulmonary dysplasia: a detailed family study. Canadian Medical Journal, 95, 603 .

Austrian, R., McClement, J. H., Renzetti, A. D. Jr., Donald, K. W., Riley, R. L., and Cournand, A. (1951). Clinical and physiologic features of some types of pulmonary diseases with impairment of alveolar-capillary diffusion: the syndrome of alveolar-capillary block. American Journal of Medicine, 11, 667.

Baldwin, E. de F., Cournand, A., and Richards, D. W., Jr. (1948). Pulmonary insufficiency. I. Physiological classification, clinical methods of analysis, standard values in normal subjects. Medicine, 27, 243.

Banaszak, E. F., Thiede, W. H., and Fink, J. N. (1970). Hypersensitivity pneumonitis due to contamination of an air conditioner. New England Journal of Medicine, 283, 271.

Barboriak, J. J., Sosman, A. J., and Reed, C. E. (1965). Serological studies in pigeon-breeder's disease. Journal of Laboratory and Clinical Medicine, 65, 600.

Bonanni, P. P., Frymoyer, J. W., and Jacox, R. F. (1965). A family study of idiopathic pulmonary fibrosis. American Journal of Medicine, 39, 411.

Bullivant, C. M. (1966). Accidental poisoning by paraquat: report of 2 cases in man. British Medical Journal, 1, 1272.

Campbell, J. M. (1932). Acute symptoms following work with hay. British Medical Journal, 2, 1143.

Corbaz, R., Gregory, P. H., and Lacey, M. E. (1963). Thermophilic and mesophilic actinomycetes in mouldy hay. Journal of General Microbiology, 32, 449.

Cotes, J. E. (1963). Terminology for exchange of gas in the lungs. Lancet, $2,843$.

Crofton, J. and Douglas, A. (1969). Respiratory Diseases. Blackwell, Oxford.

De Lena, M., Guzzon, A., Monfardini, S., and Bonadonna, G. (1972). Clinical, radiologic, and histopathologic studies on pulmonary toxicity induced by treatment with bleomycin. Cancer Chemotherapy Reports, Part I, 56, 343.

Dickie, H. A. and Rankin, J. (1958). Farmer's lung: an acute granulomatous interstitial pneumonitis occurring in agricultural workers. Journal of the American Medical Association, 167, 1069.

Donohue, W. L., Laski, B., Uchida, I., and Munn, J. D. (1959). Familial fibrocystic pulmonary dysplasia and its relation to the Hamman-Rich syndrome. Pediatrics, 24, 786.

Fawcitt, R. (1936). Fungoid conditions of the lung. British Journal of Radiology, 9, 172, 354.

Fleischman, R. W., Baker, J. R., Thompson, G. R., Schaeppi, U. H., Illievski, V. R., Cooney, D. A., and Davis, R. D. (1971). Bleomycin-induced interstitial pneumonia in dogs. Thorax, 26, 675. 
Fuller, C. J. (1953). Farmer's lung: a review of present knowledge. Thorax, 8, 59.

Grant, I. W. B., Hillis, B. R., and Davidson, J. (1956). Diffuse interstitial fibrosis of the lungs (Hamman-Rich syndrome). American Review of Tuberculosis, 74, 485.

Gregory, P. H., Festenstein, G. N., Lacey, M. E., Skinner, F. A., Pepys, J., and Jenkins, P. A. (1964). Farmer's lung disease: the development of antigens in moulding hay. Journal of General Microbiology, 36, 429.

— and Lacey, M. E. (1963). Mycological examination of dust from mouldy hay associated with farmer's lung disease. Journal of General Microbiology, 30, 75.

Hapke, E. J., Seal, R. M. E., Thomas, G. O., Hayes, M., and Meek, J. C. (1968). Farmer's lung: a clinical, radiographic, functional, and serological correlation of acute and chronic stages. Thorax, 23, 451 .

Hargreave, F. E., Pepys, J., Longbottom, J. L., and Wraith, D. G. (1966). Bird breeder's (fancier's) lung. Lancet, 1, 445.

von Hayek, H. (1953). Die menschliche Lunge. Springer, Berlin.

(1960). The Human Lung, translated by V. E. Krahl. Hafner, New York.

Heard, B. E. and Cooke, R. A. (1968). Busulphan lung. Thorax, 23, 187.

Koch, B. (1965). Familial fibrocystic pulmonary dysplasia. Canadian Medical Association Journal, 92, 801.

Liebow, A. A. (1968). New concepts and entities in pulmonary disease. In The Lung, edited by A. A. Liebow and D. E. Smith. Williams and Wilkins, Baltimore. p. 332.

—, Steer, A., and Billingsley, J. G. (1965). Desquamative interstitial pneumonia. American Journal of Medicine, 39, 369.

Littler, W. A., Kay, J. M., Hasleton, P. S., and Heath, D. (1969). Busulphan lung. Thorax, 24, 639.

Livingstone, J. L., Lewis, J. G., Reid, L., and Jefferson, K. E. (1964). Diffuse interstitial pulmonary fibrosis: a clinical, radiological, and pathological study based on 45 patients. Quarterly Journal of Medicine, 33, 71.

Mason, A. M. S., McIllmurray, M. B., Golding, P. L., and Hughes, D. T. D. (1970). Fibrosing alveolitis associated with renal tubular acidosis. British Medical Journal, 4, 596.

Matthew, H., Logan, A., Woodruffe, M. F. A., and Heard, B. (1968). Paraquat poisoning: lung transplantation. British Medical Journal, 3, 759.

Oliner, H., Schwartz, R., Rubio, F., Jr., and Dameshek, W. (1961). Interstitial pulmonary fibrosis following busulphan therapy. American Journal of Medicine, 31, 134.

Pepys, J. (1967). Hypersensitivity to inhaled organic antigens. Journal of the Royal College of Physicians, 2, 42.
Jenkins, P. A., Festenstein, G. N., Gregory, $\overrightarrow{\overline{\vec{D}}}$ P. H., Lacey, M. E., and Skinner, F. A. (1963). Farmer's lung: thermophilic actinomycetes as a source of 'farmer's lung hay' antigen. Lancet, $\underline{\bar{\sigma}}$ 2, 607.

, Longbottom, J. L., and Jenkins, P. A. (1964). Vegetable dust pneumoconioses. American $R e-0$ view of Respiratory Diseases, 89, 842.

Riddell, R. W., Citron, K. M., and Clayton, $\vec{\circ}$

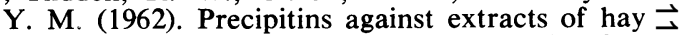
and moulds in the serum of patients with far- $\omega$ mer's lung, aspergillosis, asthma, and sarcoidosis. Thorax, 17, 366.

Riddle, H. F. V., Channell, S., Blyth, W., Weir, ஸ̂ D. M., Lloyd, M., Amos, W. M. G., and Grant, $\omega$ I. W. B. (1968). Allergic alveolitis in a malt-iv worker. Thorax, 23, 271

Rubin, E. H. and Lubliner, R. (1957). The Hamman- 윽 Rich syndrome: review of the literature and analysis of 15 cases. Medicine, 36, 397.

Sakula, A. (1967). Mushroom-worker's lung. British Medical Journal, 3, 708.

Scadding, J. G. (1952). Chronic lung disease with diffuse nodular or reticular radiographic shadows. $\stackrel{+}{+}$ Tubercle, 33, 352.

(1956). Pulmonary fibrosis and collagen diseases of the lung. British Journal of Radiology, 29, 633.

(1959). Principles of definition in medicine. Lancet, 1, 323.

(1960). Chronic diffuse interstitial fibrosis of the lungs. British Medical Journal, 1, 443.

(1964). Fibrosing alveolitis. British Medical Journal, 2, 686.

(1966). Patterns of respiratory insufficiency. Lancet, 1, 701.

(1967a). Diagnosis: the clinician and the computer. Lancet, 2, 877.

(1967b). Sarcoidosis. Eyre and Spottiswoode, $\frac{0}{3}$ London.

(1969). The lungs in rheumatoid arthritis. Pro-윽 ceedings of the Royal Society of Medicine, 62, 227.

(1970). Lung biopsy in the diagnosis of diffuse lung disease. British Medical Journal, 2, 557.

(1972). The semantics of medical diagnosis. $N$ Biomedical Computing, 3, 83.

and Hinson, K. F. W. (1967). Diffuse fibrosing $N$ alveolitis (diffuse interstitial fibrosis of the lungs): N correlation of histology at biopsy with prognosis. Thorax, 22, 291.

Seal, R. M. E., Hapke, E. J., Thomas, G. O., Meek, $\stackrel{\varrho}{\text {, }}$ J. C., and Hayes, M. (1968). The pathology of the acute and chronic stages of farmer's lung.? Thorax, 23, 469.

Staines, F. H. and Forman, J. A. S. (1961). A survey of 'farmer's lung'. Journal of the College of General Practitioners, 4, 351.

Swaye, P., van Ordstrand, H. S., McCormack, L. J. @ and Wolpaw, S. E. (1969). Familial Hamman-Rich syndrome. Diseases of the Chest, 55, 7. 
Totten, R. S., Reid, D. H. S., Davis, H. D., and Moran, T. J. (1958). Farmer's lung: report of 2 cases in which lung biopsies were performed. American Journal of Medicine, 25, 803.

Törnell, E. (1946). Thresher's lung. Acta Medica Scandinavica, 125, 191.

Turner-Warwick, M. (1968). Fibrosing alveolitis and chronic liver disease. Quarterly Journal of Medicine, 37, 133.

and Doniach, D. (1965). Auto-antibodies in interstitial pulmonary fibrosis. British Medical Journal, 1, 886.

and Haslam, P. (1971). Antibodies in some chronic fibrosing lung diseases. I. Non organspecific auto-antibodies. Clinical Allergy, 1, 83.
Wagley, P. F. (1972). A new look at the HammanRich syndrome. John Hopkins Medical Journal, 131. 412 .

West, J. B. (1965). Ventilation/Blood Flow and Gas Exchange. Blackwell, Oxford.

Williams, J. V. (1961). Inhalation tests with hay and fungi in patients with farmer's lung. Acta Allergologica, 16, 77.

(1963a). Inhalation and skin tests with extracts of hay and fungi in patients with farmer's lung. Thorax, 18, 182.

(1963b). Pulmonary function studies in patients with farmer's lung. Thorax, 18, 255.

Requests for reprints to: Professor J. G. Scadding, Brompton Hospital, Fulham Road, London SW3. 